\title{
Astroviren und Toroviren
}

\author{
H.R. Brodt
}

50.1 Astroviren - 403

50.1.1 Erreger - 403

50.1.2 Epidemiologie - 403

50.1.3 Klinik und Diagnostik - 404

50.1.4 Therapie und Präventivmaßnahmen - 404

50.2 Toroviren -404

Literatur - 405

et al. 1993). Morphologisch sind Astroviren ikosaedrische Partikel mit einem Durchmesser von 28-30 nm. Entsprechend ihrer Namensgebung erscheinen im Elektronenmikroskop etwa $10 \%$ aller Viren sternförmig. Das Virus hat ein Genom aus einer Einzelstrang-RNA mit positiver Polarität und keine Hülle, ähnlich den Caliciviridae (Matsui u. Greenberg 1996). Bis heute können 7 Serotypen unterschieden werden, bei denen sich auch unterschiedliche Genotypen nachweisen ließen (Noel et al. 1995). Die Inzidenz der Serotypen wird aus 2 Untersuchungen in Oxford und Utrecht abgeleitet, nach denen der Serotyp 1 der mit Abstand häufigste, die Serotypen 2-4 wenig häufig und die Typen 5-7 selten sind.

Astroviren können in primären und permanenten Zelllinien kultiviert werden, wenn diesen $10 \mu \mathrm{g}$ Trypsin pro ml Kulturmedium zugefügt wird (Lee u. Kurtz 1994).

in Zusammenhang mit Durct erkrankungen gebracht, ohne dass dies allerdings bis heute bewiesen werden konnte (Townsend et al. 1982). Gleiches gilt auch für Reoviren und Toroviren (Koopmans et al. 1997).

\subsection{Astroviren}

\subsubsection{Erreger}

Astroviren gehören zu der neu definierten Familie der Astroviridae von humanen und animalen Spezies (Monroe

\subsubsection{Epidemiologie}

Astroviren wurden weltweit beim Menschen, aber auch bei vielen Tierspezies wie Wild, Schweinen, Hunden, Mäusen und Enten gefunden. Es hat sich gezeigt, dass diese Viren sich sehr speziesspezifisch verhalten und mit Ausnahme der Enten (Hepatitis) bei den betreffenden Tieren wie beim Menschen Durchfallerkrankungen auslösen können. 
Menschliche Astrovirusinfektionen finden sich vorwiegend bei Kleinkindern und älteren Erwachsenen. Wie bei anderen enteritischen Virusinfektionen sind Infektionen in der Kindheit am häufigsten, wobei vermutlich die meisten Infektionen inapparent oder zumindest unbemerkt verlaufen, da sich bei etwa $75 \%$ aller 10-Jährigen Astrovirusantikörper nachweisen lassen. Astroviren wurden auch gelegentlich als Erreger von Diarrhöen nach Lebensmittelintoxikationen identifiziert (Matsui et al. 1994, Mitchell et al. 1995, Oishi et al. 1994).

Die Inzidenz von astrovirusinduzierten Diarrhöen wird im Rahmen von Studien in Krankenhäusern mit weniger als 5\% angegeben, wenn auch aus einzelnen Ländern wie Guatemala oder Thailand mit ca. 10\% höhere Inzidenzen beschrieben wurden. Aus der Zeit vor der Einführung der hoch aktiven antiretroviralen Therapie wird in einer Studie bei HIV-infizierten Patienten mit Durchfallerkrankungen die Ursache in etwa $15 \%$ in Astrovirusinfektionen gesehen (Grohmann et al. 1993). Auch die Astrovirusinfektionen treten in der westlichen Welt gehäuft in den Wintermonaten auf. Die Übertragung wird vorwiegend auf eine fäkal-orale Infektion zurückgeführt, wenn auch diese über infizierte Lebensmittel oder direkt von Mensch zu Mensch möglich ist.

\subsubsection{Klinik und Diagnostik}

Die wenigen Erkenntnisse zur Pathogenese der Astrovirusinfektion resultieren aus In-vitro-Studien mit infizierten Zellen und aus Untersuchungen an Versuchstieren. Die Absorption der Viren erfolgt vermutlich über einen zellulären Rezeptor, und die Replikation der Viren findet im Zytoplasma der Zellen statt. Bei experimentell infizierten Tieren (Schafe und Kälber) mit speziesspezifischen Astroviren kommt es 14-38 h nach Virusapplikation zum Nachweis der Infektion in den Enterozyten der Villi des Dünndarms, gefolgt von Durchfällen nach 2-4 Tagen.

Im Vordergrund der klinischen Symptomatik stehen bei Astrovirusinfektionen fast ausschließlich selbstlimitierende Durchfälle. Das klinische Bild entspricht damit weitgehend einer milde verlaufenden Rotavirusinfektion ( Kap. 47). Schwere Verlaufsformen sind kaum bekannt und nur bei immunsupprimierten Patienten zu erwarten.

Die Diagnose einer Astrovirusinfektion ist grundsätzlich durch eine aufwendige elektronenmikroskopische Untersuchung möglich. Praktisch erfolgt allerdings der Nachweis einer Infektion heute über einen kommerziell verfügbaren Antigentest auf EIA-Basis, der auch alle 7 Serotypen erkennen kann (Herrmann et al. 1990). Auf Genbasis ist auch prinzipiell der Nachweis mit Hilfe einer RT-PCR möglich (Noel et al. 1995).

\subsubsection{Therapie und Präventivmaßnahmen}

Eine spezifische Behandlung von Astrovirusinfektionen steht nicht zur Verfügung und ist auch bei dem gutartigen Verlauf der zumeist selbstlimitierenden Infektionen nicht notwendig. Auch eine symptomatische Behandlung ist selten erforderlich, nur bei Säuglingen und Kleinkindern kann eine Überwachung bzw. Sicherstellung der Hydratation notwendig sein. Aufmerksamkeit bedürfen immer Hinweise auf das vermehrte Auftreten von Durchfallerkrankungen, v. a. in Gemeinschaftseinrichtungen, die dann neben prophylaktischen Hygienemaßnahmen auch immer mit einem Versuch des Erregernachweises einschließlich Astroviren verbunden sein sollte.

Zur Vermeidung von Infektionsausbrüchen in Gemeinschaftseinrichtungen sollten akut erkrankte Patienten diese nicht besuchen oder z. B. im Krankenhaus, wenn möglich, isoliert werden. Die vielfältigen möglichen Übertragungswege von Astroviren und den oben genannten anderen Virusspezies können dennoch bei den üblichen hygienischen Vorsichtsmaßnahmen nicht immer Infektionen verhindern, auch weil die Viren gegenüber üblichen Desinfektionsmitteln auf der Basis von Alkohol und Äther zumeist unempfindlich sind und auch das normale Händewaschen nicht immer zu einer Elimination der Erreger führt. Die Verwendung von Einmalhandschuhen sollte im Umgang mit möglicherweise kontaminierten Materialien im Krankenhaus obligat sein.

\subsection{Toroviren}

Toroviren sind einstrangige RNA-Viren mit einer Hülle, die enterale Infektionen bei Tieren hervorrufen (Hamer u. Gorbach 2002). Andere Untersuchungen haben jedoch ergeben, dass Toroviren auch Diarrhö bei Kindern bewirken können (Jamieson et al. 1998, Koopmans et al. 1997). In einer prospektiven Studie über virale Diarrhöen bei Kindern fanden sich zu 3\% Toroviren, die damit häufiger vertreten waren als Calici- oder Astroviren (Waters et al. 2000). Die meisten Diarrhöen durch das Torovirus kommen bei Kindern unter dem Alter von 2 Jahren vor.

Das Torovirus vermag sowohl akute wie auch chronische Diarrhöen (Dauer >14 Tage) zu bewirken (Glass et al. 1996, Jamieson et al. 1998, Koopmans et al. 1997). Bei Kindern mit anhaltender Diarrhö durch das Torovirus fanden sich auch Koinfektionen, z. B. mit enteroaggregativen Escherichia coli (EaggEC) (Hamer u. Gorbach 2002).

Klinisch kommt Erbrechen seltener als bei der Rotavirusinfektion vor, die Stühle können jedoch auch blutig sein. Das Torovirus ist im Stuhl durch EM oder ELISA nachweisbar.

Die Therapie ist symptomatisch und besteht ebenfalls in der oralen oder parenteralen Rehydratation, die oft über eine ganze Woche erfolgen muss (Jamieson et al. 1998). 


\section{Literatur}

Chang C, Moscovici O, Lebon P et al. (1982) Association of coronavirus infection with neonatal necrotizing enterocolitis. Pediatrics 69 : 209-214

Glass Rl, Noel J, Mitchell D et al. (1996) The changing epidemiology of astrovirus-associated gastroenteritis: a review. Arch Virol Suppl 12: $287-300$

Grohmann GS, Glass RI, Pereira HG et al. (1993) Enteric viruses and diarrhea in HIV-infected patients. N Engl J Med 329: 14-20

Hamer DH, Gorbach SL (2002) Infectious diarrhea and bacterial food poisening. In: Feldman M, Friedman LS Sleisenger MV (eds) Gastrointestinal and liver disease, $7^{\text {th }}$ ed. Saunders, Philadelphia, pp 1864-1913

Herrmann JE, Nowak NA, Perron-Henry DM et al. (1990) Diagnosis of astrovirus gastroenteritis by antigen detection with monoclonal antibodies. J Infect Dis 161: 226-229

Jamieson FB, Wang EEL, Bain C et al. (1998) Human torovirus: a new nosocomial gastrointestinal pathogen. J Infect Dis 178: 1263-1269

Koopmans MPG, Goosen ESM, Lima AM et al. (1997) Association of torovirus with acute and persistent diarrhea in children. Pediatr Infect Dis J 16: 504-507

Lee TW, Kurtz JB (1994) Prevalence of astrovirus serotypes in the Oxford region 1976-92 with evidence for 2 new serotypes. Epidemiol Infect 112: 187-193

Matsui SM, Greenberg HB (1996) Astroviruses. In: Fields BN, Knipe DM, Howley PM et al. (eds) Fields virology $3^{\text {rd }}$ edn. Lippincott-Raven, Philadelphia, pp 811-824

Matsui SM, Lewis TL, Chiu E et al. (1994) An outbreak of astrovirus gastroenteritis in a nursing home and molecular characterization of the virus. Gastroenterology 106: A 730

Melnick JL (1996) Enteroviruses: polioviruses, coxsackieviruses, echoviruses, an newer enteroviruses. In: Fields BN, Knipe DM, Howley PM et al. (eds) Fields virology $3^{\text {rd }}$ edn. Lippincott-Raven, Philadelphia, pp 655-712

Mitchell DK, Monroe SS, Jiang X et al. (1995) Virologic features of an astrovirus diarrhea outbreak in a day care centre revealed by reverse transcription-polymerase chain reaction. J Infect Dis 172: $1437-1444$

Monroe SS, Jiang B, Stine SE et al. (1993) Subgenomic RNA sequence of human astrovirus supports classification of Astroviridae as a new family of RNA viruses. J Virol 67: 3611-3614

Noel JS, Lee TW, Kurz JB et al. (1995) Typing of human astroviruses from clinical isolates by enzyme immunoassay and nucleotide sequencing. J Clin Microbiol 33: 797-801

Oishi I, Yamazaki K, Kimoto T et al. (1994) A large outbreak of acute gastroenteritis associated with astrovirus among students and teachers in Osaka, Japan. J Infect Dis 170: 439-443

Townsend TR, Bolyard EA, Yolken RH et al. (1982) Outbreak of Coxsackie $A 1$ gastroenteritis: a complication of bone marrow transplantation. Lancet i 820-823

Waters V, Ford-Jones EL, Petric M et al. (2000) Etiology of communityacquired pediatric viral diarrhea: a prospective longitudinal study in hospitals, emergency departments, pediatric practices, and child care centers during the winter rotavirus outbreak, 1997 to 1998. Pediatr Infect Dis 19: 843-850 